\title{
Evaluation of a modified interview version and of a self-rating version of the Suicide Assessment Scale
}

\author{
A. Niméus *, F. Hjalmarsson Ståhlfors, C. Sunnqvist, B. Stanley, L. Träskman-Bendz \\ Department of Psychiatry, Clinical Sciences, Lund University Hospital, S-22185 Lund, Sweden
}

Available online 20 March 2006

\begin{abstract}
The Suicide Assessment Scale (SUAS) was constructed to be sensitive to change of suicidality. It was recently found to be predictive of suicide in a group of suicide attempters. The aim of the present study was to evaluate the reliability and validity of a modified interview version of SUAS with defined scores and also a new self-rating version (SUAS-S). The subjects consisted of former inpatients, 42 persons who had been admitted because of a suicide attempt about 12 years ago and 22 control patients. The subjects were rated according to the SUAS, the SUAS-S, as well as the Montgomery Asberg Depression Rating Scale (MADRS). The interrater reliability was found to be high. The SUAS correlated significantly with the MADRS, but the concordance was not consistent, which indicates that the SUAS measures something different from depression. The SUAS-S correlated significantly with the interview-rated SUAS, thus exhibiting good concurrent validity. In summary, both the modified interview version of SUAS and the SUAS-S seem to be valid, reliable and easily used suicide assessment instruments.
\end{abstract}

(C) 2006 Elsevier Masson SAS. All rights reserved.

Keywords: Rating scale; Suicide Assessment Scale (SUAS); Suicidality; Suicide prediction

\section{Introduction}

Whether or not a patient is likely to kill him/herself is a delicate and crucial assessment in every day psychiatric practice. Decisions such as the type of treatment and if a patient should be admitted to institutional care lean heavily on this evaluation. There are different methods of suicide risk assessment, e.g. an unstructured clinical interview or a structured clinical interview using a rating scale. Especially after a suicide attempt, Hawton [1] recommended a semi-structured assessment, possibly combined with questionnaires. He not only emphasized the importance of facing the actual problems, but also taking into consideration the risk of further suicidal behavior.

As discussed by Pokorny [2] and Hawton [3], there are difficulties involved in making statements about the suicide risk of an individual. Among these is the fact that suicide is a rare event, which could result in high rates of false positive prediction [4]. Other drawbacks are differences between short term

\footnotetext{
* Corresponding author. Tel.: +46 4617 3845; fax: +46 46173840 .

E-mail address: anders.nimeus@med.lu.se (A. Niméus).
}

and long term predictors [5]. Furthermore there is a limited evidence that suicide risk screening among unidentified primary care patient improves the outcome [6]. One approach to assessment of suicide risk in psychiatric care is the use of rating scales related to suicidality. One such scale is the Suicidal Intent Scale [7], which is interview based. This scale has lately been used in several studies, and high scores in the entire scale, or parts of it, might be predictive of suicide in different psychiatric populations [8-11]. In one study, Hjelmeland et al. [12] were not able to find SIS-differences between suicides and non-suicides among suicide attempters. The self-rated Beck Hopelessness Scale (BHS) measuring pessimism towards the future does however not seem to predict suicide in suicide attempters, but rather to be related to ratings of depression [13]. Suicide items are also included in depression rating scales, such as the Montgomery Asberg Depression Rating Scale (MADRS) [14] and the Hamilton Depression Rating Scale (HDRS) [15]. The Suicide Assessment Scale (SUAS) was developed by Stanley et al. [16] to assess changes in suicidality over time and preferably during treatment. Three criteria were considered: 1 . To measure both observable and reported symptomatology associated with suicidality. 2 . Not to be linked to a 
specific diagnosis. 3. To be sensitive to change over time [16]. In the original version, the item-scores were without specific definitions. Originally, both validity and reliability was good, showing significantly higher scores among suicide attempters than non-attempters (criterion validity), producing significant correlations with already established rating instruments (concurrent validity), and an interrater reliability varying between 0.78 and 0.88 [16]. In a group of hospitalized suicide attempters we [17] carried out a prospective study on the predictive validity of this scale, and found high SUAS scores to predict suicide within 1 year after a suicide attempt. A cut-off score, indicating a high risk of suicide within a year, was established. Concurrent validity was again found to be good. An interrater reliability test was however not performed. In a recent study by members of our research group high SUAS scores were associated with low cerebrospinal fluid (CSF) 5-hydroxyindoleacetic acid (5-HIAA) and non-suppression of cortisol (dexamethasone suppression test) [18], which are two presumptive biological markers of suicidality.

Self-rating scales could improve the efficiency of the clinical examination. Joiner et al. [19] compared self- versus clinician-rated suicide assessment in patients who presented with suicidal thoughts or behavior. The patients rated themselves as less suicidal than did the clinicians. Furthermore, the self-ratings offered a good predictive value. Prusoff et al. [20] showed that self-reports of depressive symptoms were not reliable in the acute episode, but concorded better with clinical assessment at follow-up. Self-rating scales would therefore be of value when evaluating recovery from depression.

The aims of this study were:

- to calculate the interrater reliability of a modified SUAS;

- to evaluate the concurrent validity of a self-rating version of SUAS (SUAS-S);

- to evaluate whether SUAS reflects suicidality rather than depression.

\section{Subjects and methods}

\subsection{Sample}

We used an already available material of 42 patients taking part in a 12-year follow-up study after a suicide attempt and 22 control patients, totally 64 subjects.

The former suicide attempters (21 men; 21 women) had a mean age of $51.0 \pm 10.1$ years; median 50.5

The 22 controls (nine men; 13 women) were matched with 22 of the former suicide attempters according to time of inpatient care, diagnosis, age and sex but had not made a suicide attempt before the index admission to hospital. Their mean $\pm \mathrm{S}$. D. age was $49.8 \pm 8.5$ years; median 50.5 . In this study the two groups are combined.

\subsection{Ratings}

The subjects were rated according to the MADRS and the modified interview version of SUAS (see below). The participants were also offered to use the recently constructed SUAS self-rating scale (SUAS-S).

Both kinds of SUAS are numeric scales, consisting of 20 items, each item rated in terms of severity $(0-4$ points). The items are grouped into five areas: affect, bodily states, control and coping, emotional reactivity, and suicide thoughts and behavior. The expert interview version takes about 20-30 min to fill in. Contrary to the original SUAS, the modified version contains defined scores, which should make the scale easier to use [15] and thereby improving interrater reliability.

The MADRS is an interview-based depression scale designed to be sensitive to change in severity of depression [14]. The original MADRS consists of 10 items each scored $0-3$ with half-points (a later version includes full points, and the scores thus range from 0 to 6 ). The MADRS contains one item which evaluates suicide thoughts.

The SUAS-S (self-rating) was filled in by each patient before the interview-rating, and the interviewers were not aware of the self-rating results beforehand. The SUAS and MADRS interview-ratings were carried out simultaneously by a senior psychiatrist and a resident. Co-training of ratings preceded the study. Since the residents had other duties they only rated 42 patients. Three of the 64 patients taking part failed to complete the SUAS-S.

\subsection{Psychiatric diagnoses}

Diagnoses were made according to the DSM IV [21] by the senior psychiatrist. Diagnoses were settled independently of SUAS-ratings.

\subsection{Statistics}

The Statistical Program for Social Sciences (SPSS) [22] was used to perform the statistics. Non-parametric statistics were used when the number of observations was small $(<25)$, or not normally distributed and also because SUAS is a nominal scale $[15,17]$. The Mann-Whitney $U$-test was used to detect significant differences. For correlations Spearman rank correlation coefficients were utilized.

Only the senior psychiatrist ratings were used in the calculations relating SUAS interview-ratings to diagnostic groups or the MADRS.

\subsection{Informed consent and ethics committee}

All patients gave written informed consent and the Lund University Medical Ethics Committee had approved the study. 


\section{Results}

\subsection{Sample characteristics}

In total, 64 individuals were studied. There were $30(47 \%)$ men (mean age $51.2 \pm 7.8$ years, range 35-65) and 34 (53\%) women (mean age $51.2 \pm 10.9$ years, range $34-78$ ). No significant difference in age between men and women existed (Mann-Whitney, NS).

\subsection{Diagnoses}

Patients could have more than one DSM IV axis I diagnosis. However, in this study only the principal diagnosis was considered. Major depressive disorder (MDD) was the predominant diagnosis $(N=14,21.9 \%)$, but lack of an axis I diagnosis was even more common $(N=29,45.3 \%)$ (Table 1$)$.

\subsection{Ratings}

\subsubsection{Age and gender}

There were no significant differences in interview SUAS scores between men and women (mean $6.6 \pm 10.5$; median 2.0 vs. $9.1 \pm 11.3$; median 4.0, Mann-Whitney, NS). Similarly, no significant SUAS differences (but a strong tendency) were found between subjects with high $(N=32)$ and low $(N=32)$ age, respectively, defined as above or below the mean/median age of 51 years (mean $5.8 \pm 10.3$; median 1.0 , vs. $10.1 \pm 11.3$; median 4.5, Mann-Whitney, $P=0.05$ ).

Table 1

Distribution of DSM IV axis I-diagnoses by sex

\begin{tabular}{llll}
\hline & Men & Women & Total \\
\hline 0 & 14 & 15 & 29 \\
MDD & 6 & 8 & 14 \\
Dysthymia & 1 & 3 & 4 \\
Alcoholism & 3 & 2 & 5 \\
Anxiety disorders & 1 & 2 & 3 \\
Psychotic disorders & 2 & 0 & 2 \\
Other diagnosis & 3 & 4 & 7 \\
Total & 30 & 34 & 64 \\
\hline
\end{tabular}

\subsubsection{Diagnoses}

The distribution of SUAS scores by diagnoses is tabulated in Table 2. In all but one diagnostic group, psychosis $(N=2)$, self-ratings generated higher scores than interview ones.

Since the number of subjects was small, the interview based SUAS scores of those who had received an axis I diagnosis $(N=35)$ were compared with those without $(N=29)$. It turned out to be significantly higher in those with than without a diagnosis $(11.1 \pm 12.3$, median 4.5 vs. $1.8 \pm 2.1$, median 1.0 , Mann-Whitney, $P<0.01)$. There were no significant differences between people suffering from a mood disorder $(N=18$, mean score $11.7 \pm 13.9$, median 4.0) compared to people with some other axis I diagnosis $(N=17$, mean score $11.6 \pm 11.0$, median 11.0) (Mann-Whitney, NS).

\subsection{Correlation between SUAS and MADRS}

The correlation between total scores of MADRS $(N=64)$ and the interview version of the SUAS $(N=64)$, respectively, the SUAS-S $(N=61)$ was 0.83 and 0.78 (Spearman's rho, $P<0.01)$.

\subsection{Interrater reliability}

Interrater reliability was measured by correlating expert ratings with resident ratings from the same interviews $(N=42)$. It turned out to be significant (Spearman's rho $=0.97, P<0.01$ ). The results of individual items varied and had a median of 0.92 .

\subsection{Validity of the self-rating scale}

The validity of the SUAS-S was tested by correlating the score on this scale with the score given by a senior psychiatrist using the SUAS interview version $(N=61)$. A significant correlation (Spearman's rho $=0.82, P<0.01$ ) was found between the total scores of these two scales. The correlations between individual items varied and had a median of 0.59 . Correlations between individual items were less significant in item 10 (Somatic concern) (Spearman's rho $=0.32, P<0.05$ ), and missing in item 11 (Impulsivity) (Spearman's rho $=0.16$, NS).

Table 2

Distribution of SUAS scores by diagnoses

\begin{tabular}{|c|c|c|c|c|c|c|c|c|c|}
\hline & & SUAS & view sc & & & & S self-ra & score & \\
\hline & $N$ & Median & Mean & S.D. & $N$ & Missing & Median & Mean & S.D. \\
\hline 0 & 29 & 1.0 & 1.8 & 2.1 & 29 & & 4.5 & 6.6 & 5.4 \\
\hline MDD & 14 & 2.5 & 7.5 & 10.9 & 14 & 1 & 8.0 & 14.2 & 13.7 \\
\hline Dysthymia & 4 & 31.5 & 28.5 & 14.0 & 4 & & 45.5 & 42.5 & 13.6 \\
\hline Alcoholism & 5 & 1.0 & 4.4 & 8.2 & 5 & & 11.0 & 10.4 & 4.7 \\
\hline Anxiety disorder & 3 & 11.0 & 12.3 & 8.1 & 3 & 1 & 23.0 & 23.0 & 5.7 \\
\hline Psychosis & 2 & 24.0 & 24.0 & 12.7 & 2 & & 14.0 & 14.0 & 15.6 \\
\hline Other diagnosis & 7 & 12.0 & 11.8 & 10.8 & 7 & & 23.0 & 19.7 & 13.3 \\
\hline Total & 64 & 2.0 & 7.9 & 11.0 & 64 & 2 & 9.0 & 13.9 & 13.6 \\
\hline
\end{tabular}

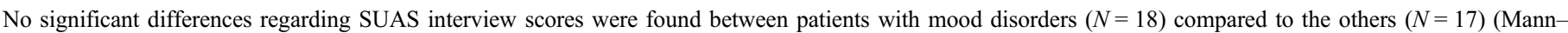
Whitney, NS). 


\section{Discussion}

This study was aimed at further evaluating a modified version of the SUAS, a scale originally designed by Stanley et al. [16]. The SUAS had originally shown good validity and a reasonably good interrater reliability [16]. Our group [17] found the SUAS alone to have a high predictive validity for suicide in a population of hospitalized suicide attempters. We however recommended a combination of DSM diagnostic procedures with the SUAS and also to take high age into consideration. This is in line with Hawton's [1] recommendations of semistructured interview including questionnaires in this complicated situation.

The SUAS was modified by us by adding defined scores to each item. Defined scores in a scale tend to make ratings less dependent on the expertise of the rater, compared to scales using non-defined scores [15]. To this date however, no interrater reliability test of the modified SUAS has been done. In addition to determining the interrater reliability of the interview-scale, the validity of our recently developed self-rating scale of the SUAS was tested.

The SUAS-S total score was found to correlate significantly with total scores of the interview-scale of SUAS, thus exhibiting concurrent validity in this research-design. It is generally known that self-ratings generate higher scores [15] than interview ratings which was also the case in our study. The main explanation for this is the tendency of patients to assess their emotions as more serious than the psychiatrist [15]. As a comparison, Joiner et al. [19] however reported that clinicians evaluated patients as more suicidal than was evident from self-ratings. One item (11, "Impulsivity") failed to show any significant correlation. Again, this finding could be explained by vague definitions and/or choice of words or by the sources of error commonly seen in self-ratings, as mentioned above.

Like our previous findings [17] the SUAS correlated significantly but not optimally, with the MADRS. Both the SUAS and the MADRS have items concerning sadness, anxiety, lack of emotions, fatigue and suicidal thoughts. The SUAS includes further items related to suicidality, so therefore a perfect concordance cannot be expected. This result combined with the finding that individuals with a principal axis I diagnosis score higher on the SUAS than those with no such diagnosis could be taken as proof of the criterion validity of the SUAS. A suicide is not only a result of depressive illness but can be found in all psychiatric illnesses.

The next step would be to test the ability of both versions (interview and self-report) to measure suicidality both shortly after a suicide attempt and at several points of time later. Such repeated ratings are the prerequisite of evaluating the SUAS as an instrument sensitive to change and its capacity of assessing recovery. The SUAS has so far only been used after a suicide attempt. Therefore another step is to test the SUAS-S as a screening instrument for suicide risk in an extended psychiatric outpatient population. It can already be concluded that both the SUAS and the SUAS-S seem to be valid and reliable suicide rating scales, which might aid the clinician in the assessment of suicide risk and also be of value in suicide research.

\section{Appendix}

\section{Manual:}

- The Suicide Assessment Scale (SUAS) in which scores of each item are defined, is available in both an interview- and a self-rating version in Swedish. The latter has recently been translated into English and Norwegian.

- So far, different versions of the SUAS (scored 0-80) have only been studied in selected psychiatric populations. Therefore, we recommend the scale to be used for research purposes, only.

\section{$S U A S-S$}

Self-rating version of the SUAS

Stanley et al., Psychopharmacology Bulletin (1986), 1, 201205.

Swedish version by Anders Niméus, MD, PhD.

Retranslated into English by professor Lil Träskman-Bendz Revised by professor Keith Newton.

\section{Instructions}

Please read carefully the groups of statements given below. Then mark the one statement in each group which best reflects your feelings during the last week-including today. Please read all the statements in each group before you make your choice. You might feel that all the statements in a group do not apply to you -in that case, please mark "0".

\section{1}

0 . I can feel both joy and sadness, according to the circumstances.

1. I am mostly positive and cheerful, but sometimes I have periods of despair.

2. I am often depressed, but can have bright periods.

3. I almost always feel depressed and miserable; better moments are rare.

4. My life is totally destroyed by the deepest despair and distress.

2

0. I seldom feel irritable.

1. I feel angry and irritable more than is usual for me.

2. I often feel angry and irritable without any real reason.

3. I almost always feel irritated and sometimes really angry without any real reason. 
4. I always feel very angry and irritable without any immediate cause.

I can hardly keep my temper.

3

0. I am able to do my everyday duties without unusually getting tired.

1. I manage my daily activities, but often get tired.

2. Sometimes I have difficulty doing my everyday duties, and often I must take a break and rest.

3. I am almost always very tired and worn out and often I cannot do my everyday duties.

4. Due to extreme tiredness, I am totally unable to do anything.

4

0. I am not sensitive to being told off or criticized.

1. Occasionally, I might feel rejected or take things personally when someone tells me off or criticizes me.

2. I feel rejected or humiliated more easily than usual when I am told off or criticized.

3. I very often feel deeply hurt and offended when I am told off or criticized.

4. People around me deliberately try to hurt me by telling me off and criticizing me.

5

0 . I keep in close and regular contact with my friends and family.

1. I keep good contact with my friends and family but less often than before.

2. Currently, I keep contact with only a few of my closest friends and family members.

3. I only talk with one or two friends from time to time.

4. I cannot stand contact with other people and I live totally on my own.

6

0. I am able to cope with emotional problems adequately.

1. Occasionally I have difficulties in handling emotional problems.

2. I have a limited ability to see how I can solve my emotional problems.

3. I seldom or never see how to overcome my emotional problems.

4. I am unable even to think about emotional problems.

7

0 . I trust myself and my decisions.
1. I am sometimes uncertain about my ability to cope with my problems.

2. I am more often at the mercy of fate or those around me than to my own ability to cope with things.

3 . I very seldom make my own decisions; on the contrary, I am at the mercy of fate or those around me.

4. I am totally controlled by faith or my surroundings and do not have any control over my own life.

8

0 . I feel relaxed.

1. It is more difficult for me to relax than usual.

2. My whole body is often unpleasantly tense.

3. I am hardly ever relaxed. I feel much muscular tension and other physical discomforts.

4. I am never relaxed but suffer all the time from severe and painful muscular tension.

9

0 . I feel calm and without worries.

1. I get worried more easily than usual.

2. I easily get worried and anxious and exaggerate my worries. However, calm moments predominate.

3. I seldom feel calm, but worries and fear about what will happen today and in the future make me anxious.

4. I constantly suffer from severe anxiety and feelings of uneasiness and I am overwhelmed by fear.

10

0. I feel physically healthy.

1. Sometimes I have worries about being physically ill. However, I can easily overcome these concerns.

2. I often think and worry about my physical health. Sometimes other people have to help me by reassuring me.

3. I am fairly convinced that I am physically ill.

4. I am convinced that I have a serious physical illness but people do not believe me. In spite of having mentioned this to other people, nobody cares.

\section{1}

0. I satisfy my wishes and needs but only after full consideration of the consequences.

1. Occasionally, I act without considering the consequences.

2. I often have difficulty in stopping acting on impulse. However, the thought of possible consequences might stop me.

3. I nearly always act according to wishes and needs, mostly without thinking of the possible consequences.

4. All I do occur on the spur of the moment and I do not care about the consequences. 
12

0 . I am very self-confident.

1. Occasionally I lack confidence but am usually able to overcome this.

2. In spite of mostly being confident, I often have a sense of failure or feel uncertain about my abilities.

3. I almost always feel worthless, and I am doubtful that things will change for the better.

4. I am good for nothing and I cannot see anything improving.

13

0 . My future looks bright.

1. Occasionally I feel pessimistic about the future.

2. I often feel pessimistic about the future, and rarely have positive thoughts.

3. I always have dark and pessimistic thoughts about my future. I hardly ever have positive thoughts.

4. I feel totally hopeless and despondent and feel that anything negative can happen to me.

14

0. I am interested and involved in things going on around me.

1. Sometimes I find it difficult to be interested in those around me.

2. I often find it very difficult to take an interest in those around me.

3. Most of the time I feel totally indifferent and uninterested even concerning my close friends and family members.

4. I am tormented by my total lack of interest in other people, even those closest to me.

15

0 . I only get annoyed or frustrated for a very good reason.

1. Very occasionally, I become irritated or frustrated for minor reasons.

2. I have quite often been annoyed and frustrated for minor reasons.

3. I very often get irritated or frustrated and often without any good reason.

4. I am always annoyed or frustrated without any reason whatsoever.

16

0 . I have quite a lot of reasons for living.

1. Sometimes I have negative thoughts about the meaning of life but I am convinced that I will continue to live.
2. Repeatedly I am unsure of my wish to live. However, reasons for living are more predominant.

3. My reasons for continuing to live are few and uncertain, and I feel pessimistic about the future.

4. I see no reason for continuing to live.

17

0 . I have no wish to die.

1. I occasionally think about dying but my wish to live is strong.

2. I sometimes think about dying and feel it would be a relief.

3. To me, death means something positive, and my will to live is weak.

4. I long for death and wish I were dead.

18

0 . I have no suicidal thoughts.

1. I have occasionally thoughts of suicide.

2. On several occasions I have experienced suicidal thoughts.

3. Very frequently I have thoughts of suicide.

4. I constantly think about suicide.

\section{9}

0 . I have no suicidal ideation.

1. If I committed suicide, it would be a revenge for old injustices. However, I have better solutions.

2. Supposing I commit suicide it should make a great stir among people.

3. If I committed suicide it would solve serious problems where no better alternatives are available.

4. A suicide means a long-desired relief and rest for both myself and my surroundings.

0 . I neither think about or plan for suicide.

1. Sometimes I have thoughts of suicide.

2. Sometimes I have thought about different methods of committing suicide, but I have no carefully prepared plans.

3. I have well thought-out plans of killing myself but I have not made any immediate preparations.

4. I am ready to kill myself and I am only waiting for the right opportunity.

\section{References}

[1] Hawton K. General hospital management of suicide attempters. In: Hawton $\mathrm{K}$, van Heeringen $\mathrm{K}$, editors. Handbook of suicide and attempted suicide. New York: John Wiley \& Sons, Ltd; 2000. p. 519-37. 
[2] Pokorny AD. Prediction of suicide in psychiatric patients. Arch Gen Psychiatry 1983;40:249-57.

[3] Hawton K. Assessment of suicide risk. Br J Psychiatry 1987;150:145-53.

[4] Beck AT, Brown GK, Steer RA, Dahlsgaard KK, Grisham JR. Suicide ideation at its worst point: a predictor of eventual suicide in psychiatric outpatients. Suicide Life Threat Behav 1999;29:1-9.

[5] Hawton K, Zahl D, Weatherall R. Suicide following deliberate self-harm: long-term follow-up of patients who presented to a general hospital. Br J Psychiatry 2003;182:537-42.

[6] Gaynes BN, West SL, Ford CA, Frame P, Klein J, Lohr KN. Screening for suicide risk in adults: a summary of the evidence for the U.S. Preventive services task force. Ann Intern Med 2004;140(10):822-35.

[7] Beck AT, Weissman A, Lester D, Trexler L. The measurement of pessimism. The hopelessness scale. J Consult Clin Psychol 1974;42:861-5.

[8] Niméus A, Alsén M, Träskman-Bendz L. High suicidal intent scores indicate future suicide. Arch Suicide Res 2002;6:211-9.

[9] Beck AT, Steer RA. Clinical predictors of eventual suicide: a 5- to 10year prospective study of suicide attempters. J Affect Dis 1989;17:203-9.

[10] Suominen K, Isometsa E, Ostamo A, Lonnqvist J. Level of suicidal intent predicts overall mortality and suicide after attempted suicide: a 12year follow-up study. BMC Psychiatry 2004;4(1):11.

[11] Harriss L, Hawton K, Zahl D. Value of measuring suicidal intent in the assessment of people attending hospital following self-poisoning or selfinjury. Br J Psychiatry 2005;186:60-6.

[12] Hjelmeland H, Stiles TC, Bille-Brahe U, Ostamo A, Salander-Renberg E, Wasserman D. Parasuicide: the value of suicidal intent and various motives as predictors of future suicidal behaviour. Arch Suicide Res 1998;4: 209-25.
[13] Niméus A. Hopelessness and suicidal behavior. J Affect Disord 1997;42: 137-44.

[14] Montgomery SA, Åsberg M. A new depression scale designed to be sensitive to change. Br J Psychiatry 1979;134:382-9.

[15] Thompson C. Descriptive dimensions of rating scales. In: Thompson C, editor. Instruments of psychiatric research. Surrey: John Wiley \& Sons, Ltd; 1989. p. 162-5.

[16] Stanley B, Träskman-Bendz L, Stanley M. The suicide assessment scale: a scale evaluating change in suicidal behavior. Psychopharm Bull 1986; 1:200-5.

[17] Niméus A, Alsén M, Träskman-Bendz L. The suicide assessment scalean instrument assessing suicide risk of suicide attempters. Eur Psychiatry 2000;15:416-23.

[18] Westrin A, Nimeus A. The dexamethasone suppression test and CSF-5HIAA in relation to suicidality and depression in suicide attempters. Eur Psychiatry 2003;18(4):166-71.

[19] Joiner Jr. TE, Rudd MD, Rajab MH. Agreement between self- and clinician-rated suicidal symptoms in a clinical sample of young adults: explaining discrepancies. J Consult Clin Psychol 1999;67(2):171-6.

[20] Prusoff BA, Klerman GL, Paykel ES. Concordance between clinical assessments and patients' self-report in depression. Arch Gen Psychiatry 1972;26(6):546-52.

[21] American Psychiatric Association. Diagnostic and statistical manual of mental disorders (4th ed.). Washington, DC. 1994.

[22] Norusis M. SPSS base system user's guide. SPSS for Windows 6.0. Chicago, USA: SPSS Inc.; 1990. 